\title{
ESTUDIO
}

\section{EL ROL DE LA ARGUMENTACIÓN EN LA ALFABETIZACIÓN CIENTÍFICA}

\author{
Antonia Larrain S.
}

Chile tiene amplios desafíos en términos de rendimiento y aprendizaje escolar. Particularmente el nivel de alfabetización científica de los jóvenes chilenos no es comparable con el nivel alcanzado por jóvenes provenientes de países desarrollados. Esto es preocupante toda vez que esta competencia es sindicada por organismos internacionales como clave para el desarrollo económico y humano del país. El presente artículo discute la promoción de la alfabetización científica en el sistema educativo subrayando el rol que tiene el discurso en el aula, y particularmente el discurso argumentativo, en esta tarea.

Palabras clave: argumentación; alfabetización científica; pensamiento; discurso; enseñanza; educación.

Antonia Larraín Sutil. Psicóloga Educacional, Ph. D. en Psicología, Pontificia Universidad Católica de Chile. Académica de la Facultad de Psicología de la Universidad Alberto Hurtado. alarrain@uahurtado.cl. 


\section{Introducción}

Chile tiene amplios desafíos en términos de rendimiento y aprendizaje escolar. Las mediciones nacionales e internacionales de calidad de la educación en las que Chile ha participado (Simce; Timss 1999, 2003; Pisa 2000, 2006; Oecd, 2000) dan pruebas fehacientes de aquello. Más aún, competencias como la alfabetización científica, sindicada por organismos internacionales (Unesco, Oecd, American Association of Advancement of Science) como clave para el desarrollo de capital humano del país y, por tanto, para su desarrollo económico, no sólo no alcanza un nivel comparable al de Estados desarrollados (Mineduc, 2004a), sino que incluso ha estado fuera de la discusión educativa y de las políticas públicas en educación básica y media en Chile.

El alfabetismo científico se caracteriza particularmente por involucrar de forma nuclear habilidades argumentativas como hipotetizar, fundamentar, plantear argumentos, anticipar posibles puntos de vista alternativos, justificar, contra-argumentar, manejar evidencia, entre otras. Esto implica que la alfabetización científica no sólo involucra una mejora en aprendizajes curriculares en ciencias sino que promueve el desarrollo de recursos cognitivos generales que facilitan la construcción flexible de conocimiento y el desarrollo de habilidades de pensamiento complejo (Kuhn, 2000; Leitão, 2000; Larraín, 2007; Masón \& Santi, 1994). Según las pocas mediciones disponibles que dan cuenta de la habilidad argumentativa de los escolares chilenos, pues no existen mediciones a gran escala que den cuenta directamente de esta habilidad en niños, se constata que ella está muy poco desarrollada. De hecho, el estudio Pisa que mide alfabetización en matemáticas, lenguaje y ciencias, define los niveles de logros más avanzados como involucrando habilidades propiamente argumentativas (Oecd, 2004; 2006) y muestra que un escaso porcentaje de la población de Chile alcanza dichos niveles de logro (Mineduc, 2004a; 2008). Este escenario, no muy alentador, fuerza a discutir y pensar seriamente cómo incorporar el desarrollo de habilidades argumentativas como tarea de la enseñanza de ciencias.

Este artículo pretende discutir la noción de alfabetización científica y el rol que juega la argumentación en ésta. La reflexión comienza discutiendo la noción misma de alfabetización científica y la relevancia del fenómeno para nuestro país. Luego se presentan algunos indicadores del estado de la alfabetización científica de los jóvenes chilenos. A 
continuación se discute la noción de argumentación con el objetivo de hacer evidente su rol en la competencia científica. Por último, se plantea el fomento de la argumentación como una tarea central del sistema educativo chileno de cara al desarrollo del pensamiento científico en la población.

\section{Alfabetización científica y sociedad del conocimiento}

\subsection{Qué es la alfabetización científica}

Se dice que la alfabetización científica es un proceso central en el desarrollo de las sociedades, esgrimiéndose tanto razones económicas como de exclusión e inclusión social. Por un lado se plantea que la competencia científica de la población es determinante para el desarrollo económico de un país, pues hoy la producción de riqueza va de la mano de la generación de conocimiento científico y del desarrollo tecnológico (Laugksch, 2000; Oecd, 2007). Por otro lado, al ser la competencia científica clave para el desarrollo económico, es determinante en el ámbito laboral de las personas. Tener mejores empleos y mejor calidad de vida, en algún sentido depende de este tipo de habilidad. Por último, el pensamiento científico no sólo es determinante hoy en día en el sistema económico de una sociedad, sino que es parte central del discurso público y del pensamiento social y, por tanto, incide en el desempeño social de los individuos.

Tanto los argumentos económicos como los que se inscriben en el continuo inclusión-exclusión social suponen que ser alfabeto implica desplegarse en ciertas actividades sociales e institucionales de manera flexible. Tal como aprender a nadar implica aprender a manejarse y recorrer un medio, alfabetizarse implica poder desplegarse en y con un medio de manera funcional en las actividades que los individuos deben realizar en su vida cotidiana. La analogía sirve para ilustrar algunos puntos de lo que normalmente se entiende en la literatura por ser alfabeto funcional (Larrain, 2002).

$\mathrm{Al}$ igual que cuando aprendemos a nadar, el proceso de alfabetización es largo (quizás dure toda una vida) y tiene muchas etapas. En la primera, cuando el niño comienza a moverse en el agua, lo hace comúnmente con otro ser humano, no en tanto otro organismo, sino con un otro que habla y media simbólicamente la relación del niño con 
el agua (un 'otro hablante', Haye, 2007). Además, probablemente lo hace con la ayuda de algún artefacto (objeto que ha sido diseñado para cumplir el objetivo de permitirle al niño flotar en el agua). Sólo luego de un apoyo sostenido y eficaz el niño es capaz de flotar en el agua solo con ciertos artefactos; primero un flotador que cubre todo su cuerpo y luego con lo que en Chile llamamos 'alitas', o flotadores que se ponen alrededor de la parte superior de los brazos. Por último, en alguna etapa avanzada y luego de haber estado muchas veces en el agua (cuando ya se es un usuario de ese medio) es posible que el niño comience a nadar sin ayuda física de un hablante o un artefacto (el hablante ya no necesita sujetarlo con las manos o cargarlo en su cuerpo). Sin embargo, si la ayuda del otro se detuviera en ese momento, el niño podrá nadar de cierta forma aunque probablemente no logrará nadar con técnicas avanzadas.

En el proceso de alfabetización también es imprescindible la ayuda de otro en el manejo de un medio. En este caso el otro también es un hablante y también se requieren artefactos (por ejemplo lápiz, papel, computadores, laboratorios, etc.).

A la vez, la alfabetización también es un proceso de muchas etapas. Ser alfabeto no es algo absoluto, sino que implica ocupar una posición relativa en el manejo flexible del medio en cuestión. Es decir, se es alfabeto cuando se aprende a leer, pero sólo en un sentido básico (manejo del código). Se avanza en la alfabetización cuando no sólo decodificamos sino que también entendemos lo que leemos (y podemos entenderlo, además, en diferentes sentidos); cuando luego entendemos distintos tipos de texto; y también se es alfabeto en un nuevo sentido, cuando no sólo entendemos sino que podemos crear: escribir historias, desarrollar narrativas, entre otros. Dicho esto, se entiende que la alfabetización siempre indica un nivel de desempeño o logro; es un concepto relativo.

Como tercer punto, en el caso de la alfabetización el proceso de adquirir maestría también implica el manejo de un medio; más aún, implica poder 'moverse' en ese medio. La diferencia es que cuando hablamos de alfabetización los medios no son medios físicos, sino medios sociales de pensamiento (Vygotski, [1934]/2001), es decir, medios en los que se despliegan los pensamientos que un grupo social desarrolla y sostiene. Entre éstos el lenguaje oral y el lenguaje escrito son paradigmáticos. Nos volvemos alfabetos funcionales de la mano de 
otro hablante que nos entrega importantes indicaciones de cómo usar funcionalmente ese medio para pensar (y para pensar cada vez de maneras más diversas y flexibles). Nos volvemos alfabetos asistiendo al proceso de pensamiento de ese otro a través del uso funcional de aquellos signos.

Sin embargo, hoy en día no sólo se dice que somos alfabetos del lenguaje verbal. También se dice que uno es matemáticamente alfabeto, es alfabeto digital, científico, entre muchos otros. Alfabetización hoy en día no apunta sólo a manejar el lenguaje verbal, aunque el lenguaje verbal atraviese cualquier tipo de alfabetización. Quiere decir algo más que eso.

La Oecd (2000) define el alfabetismo funcional como aquella competencia que tiene que ver con el manejo efectivo y funcional del lenguaje escrito que tiene un individuo en la vida cotidiana. Eso quiere decir que no es sólo el manejo del código en términos de codificar y decodificar, sino cómo los individuos se manejan en ciertas actividades sociales con ese sistema semiótico (Larrain, 2002).

Desde esta perspectiva el alfabetismo científico tampoco tiene que ver con el manejo de un código en particular. Ser alfabeto en esta área involucra el manejo del lenguaje verbal oral, escrito, pero también el matemático y, al menos en algunos niveles, el físico, químico, biológico, etc. El alfabetismo científico supone poder desempeñarse no en un sistema semiótico sino en muchos a la vez. Mejor dicho implica desempeñarse en una combinación de sistemas semióticos que confluyen de acuerdo al tipo de forma de hablar y pensar propia de la actividad científica. Desde Bajtín (2002) uno diría que la actividad científica es un género discursivo que moviliza una combinación de sistemas semióticos (habla, escritura, aritmética, álgebra, etc.) y tipos discursivos (argumentativo, narrativo), conformando una estructura simbólica funcional particular.

De esta manera, tal y como lo plantea la Oecd $(2004,2006)$ en los marcos teóricos para la evaluación de las pruebas Pisa 2003 y 2006, el alfabetismo científico no sólo involucra el tener conocimientos científicos sino la capacidad de aplicarlos funcionalmente. Esta capacidad involucra habilidades (por ejemplo, discursivas y de pensamiento) y también actitudes. De hecho se define la competencia científica como haciendo referencia a 
los conocimientos científicos de un individuo y al uso de ese conocimiento para identificar problemas, adquirir nuevos conocimientos, explicar fenómenos científicos y extraer conclusiones basadas en pruebas sobre cuestiones relacionadas con la ciencia. Asimismo, comporta la comprensión de los rasgos característicos de la ciencia, entendida como un método del conocimiento y la investigación humanas, la percepción del modo en que la ciencia y la tecnología conforman nuestro entorno material, intelectual y cultural, y la disposición a implicarse en asuntos relacionados con la ciencia, y con las ideas de la ciencia como un ciudadano reflexivo. (Oecd, 2006, p. 13)

Dicho lo anterior, se entiende que el alfabetismo científico no es sólo tener conocimientos de ciencia, ni actitudes ni habilidades para lo científico. Tampoco es la suma de todo lo anterior. Ser alfabeto o competente científicamente significa poder pensar lo científico de cara a comprender el mundo natural y tomar decisiones con respecto a éste, es decir, movilizar conocimiento, actitudes y habilidades para desempeñarse en distintas actividades. La actividad y sus particulares demandas es lo que la pone en relación funcional con todos estos aspectos. No basta enseñar conocimientos y actitudes. Hay que preparar a las nuevas generaciones en las claves de la estructura funcional que caracteriza ese género discursivo.

\subsection{Por qué es relevante para un país}

Aunque muchos autores discuten lo que significa el alfabetismo científico, en general todos enfatizan la relevancia de esta competencia para la vida actual (Sjфberg, 1997; Laugksch, 2000). Las ciencias han sido definidas como disciplinas clave para el desarrollo económico de los países en la medida que en éstas se juega la generación de conocimiento, innovación y tecnología (Conferencia Mundial de la Ciencia de Budapest, junio-julio de 1999; Laugksch, 2000). A la vez, la innovación y generación requieren de un manejo complejo de los sistemas semióticos propios de la disciplina en orden de poder crear. La creación no es mera imaginación y divergencia, requiere de un uso lúdico de los lenguajes involucrados, es decir, un manejo flexible y reflexivo de sistemas semióticos específicos. En este sentido, tener capital humano alfabetizado en los sistemas semióticos propios de las ciencias parece ser relevante en términos económicos para los países. 
$\mathrm{Al}$ respecto, se puede esgrimir que no es necesario que toda la población se inscriba en los mayores niveles de logro de la competencia científica, pues sólo una elite es suficiente para la tarea de crear valor a través de la tecnología. Sin embargo, la ciencia en tanto género discursivo atraviesa muchas actividades cotidianas en las sociedades industrializadas, y por tanto, para participar competentemente en ellas se requiere de un manejo, al menos mínimo, de pensamiento científico.

En esta línea, estos autores plantean la importancia de esta competencia para la sociedad en general (Sjфberg, 1997; Laugksch, 2000), enfatizando que es necesaria para la vida cotidiana de todos los ciudadanos, pues es fundamental en la toma de decisiones cívicas y personales (por ejemplo, tomar medicamentos, hacer dieta, opinar acerca de temas ecológicos, entre otros).

Es por esto que aunque el estudiante no se dedique a las ciencias en su futura vida laboral, el desarrollo de pensamiento científico es crucial para el desarrollo humano, político y económico de un país (Mineduc, 2004a; Millwood, 2006; Erdunen, Ardac \& Yakmaci-Guzel, 2006). Estas competencias determinarán tanto la oportunidad que tendrá un país de competir en términos de innovación y generación tecnológica, como la oportunidad que tendrá un joven de incorporarse al mundo social (a través del manejo de formas de pensamiento predominantes).

Siendo una competencia tan relevante para muchos autores y encargados de definir las políticas públicas educativas, llama la atención la poca discusión que ha existido en nuestro país acerca del alfabetismo científico y su distribución en la población a nivel escolar. Aunque no hay demasiadas declaraciones públicas al respecto, las ideas que emplazan la necesidad de empujar el desarrollo de esta competencia son coherentes con el ideario que hoy predomina en el discurso público educativo (más específicamente con respecto a la educación superior y el impulso en la formación de posgrados). Por algo el país ha estado interesado en medirse internacionalmente para saber cómo está distribuida la competencia científica en los jóvenes chilenos que actualmente están en el sistema educativo. Es importante saber cómo estamos en Chile para proyectar qué hacemos por avanzar al respecto. A continuación se describirá brevemente lo primero para luego detenernos en levantar ciertas ideas que orienten lo segundo. 


\subsection{La alfabetización científica en Chile}

En Chile, el aprendizaje de los contenidos curriculares en ciencia en comparación con países desarrollados es muy bajo. Según los resultados del Estudio Internacional de Tendencias en Matemática y Ciencias (Timss) 1999, prueba que mide el manejo de contenidos curriculares en ciencia y matemáticas en octavo básico y en la que Chile participó, sólo el 1\% de los estudiantes chilenos de octavo básico muestra que es capaz de manejar los contenidos curriculares de enseñanza básica cabalmente, el $28 \%$ sólo exhibe un manejo elemental y el $44 \%$ demuestra un manejo menos que elemental de la ciencia (Eyzaguirre y Le Foulon, 2001).

En el caso del Timss del año 2003 (Mineduc, 2004b) el 44\% de los estudiantes chilenos sólo muestra un conocimiento científico inferior al mínimo que permite describir la misma prueba, y el 51\% está entre el nivel de logro inferior y el intermedio (32\% y 19\% respectivamente), es decir, tienen conocimientos básicos de las ciencias biológicas y físicas. Por último, sólo el 5\% de los estudiantes chilenos de octavo básico está entre los niveles de logro alto y avanzado ( $4 \%$ y $1 \%$ respectivamente), es decir demuestra comprensión conceptual de ciclos, sistemas y principios científicos y/o demuestra dominio de éstos de manera compleja y abstracta. Esto es preocupante, pues Chile está muy por debajo del promedio internacional de porcentaje de estudiantes en cada nivel de logro. En promedio, el 23\% de estudiantes de octavo básico de los distintos países participantes en la prueba se encuentra bajo el nivel más bajo descrito; el $24 \%$ sólo alcanza el nivel bajo, el 29\% sólo el nivel intermedio y el 19\% y $5 \%$ el nivel alto y avanzado respectivamente. Sólo dos países tienen porcentaje de estudiantes con logros inferiores al mínimo que Chile: Filipinas (58\%) y Sudáfrica (87\%).

Más allá de los contenidos curriculares, Chile ha participado en dos versiones de la prueba internacional Pisa (2000-2006). Esta prueba mide el grado de alfabetización de jóvenes de 15 años en lenguaje, matemáticas y ciencias. Cada prueba pone énfasis en un área. En el 2000 el énfasis estuvo puesto en evaluar alfabetización lingüística mientras el 2006 en alfabetización en ciencias. Según estas mediciones, el nivel de alfabetización en ciencias de los estudiantes chilenos de 15 años, es decir, el grado en que los estudiantes muestran una comprensión y manejo flexible de la información científica, también deja que desear. El Gráfico $\mathrm{N}^{\circ} 1$ señala que en ambas mediciones en la escala de 
GRÁFICO No 1: $\quad$ PROMEDIO DESEMPEÑO DE CHILE Y PAÍSES OCDE EN ESCALA DE CIENCIAS PISA 2000 Y PISA 2006

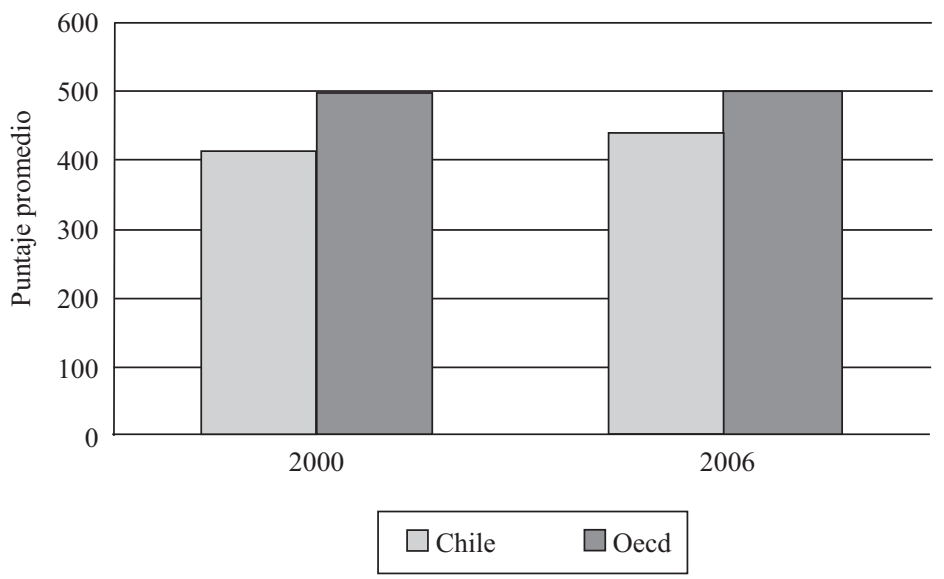

Fuente: Elaboración propia en base a Mineduc (2001) y Mineduc (2008).

ciencias los jóvenes chilenos en promedio se ubican muy por debajo del promedio de países Oecd en esa misma escala (Mineduc, 2004a).

Si comparamos ambas mediciones hay noticias positivas, pues se aprecia una mejora de los resultados en la escala de ciencias en la prueba 2006 con respecto al año 2000, y en general Chile aparece liderando los países de la región (Uruguay, Argentina, Brasil, Colombia) en la escala completa y en las tres subescalas que dan cuenta de competencias: identificación de hechos científicos; explicación científica de fenómenos; uso de evidencia científica. Sin embargo la medición de 2006 muestra que aún tenemos mucho por recorrer. Según el Gráfico $\mathrm{N}^{\circ}$ 2, prácticamente el $13 \%$ de los jóvenes que rindieron la prueba se encuentra bajo el nivel 1 de desempeño (obtiene menos de 334,9 puntos). Lo importante es que estos estudiantes, según Oecd (2007), son incapaces de demostrar las competencias requeridas por las tareas científicas más sencillas, y/o ${ }^{1}$ no pueden aplicar conocimien-

${ }^{1}$ La escala general de ciencias no implica necesariamente que los jóvenes no sean competentes en todas las subescalas. Sin embargo, en todas las subescalas más del $11 \%$ de los jóvenes se encuentra en este nivel de competencia, siendo dramático en la subescala de uso de evidencia científica, donde ese porcentaje se eleva al $16 \%$. 

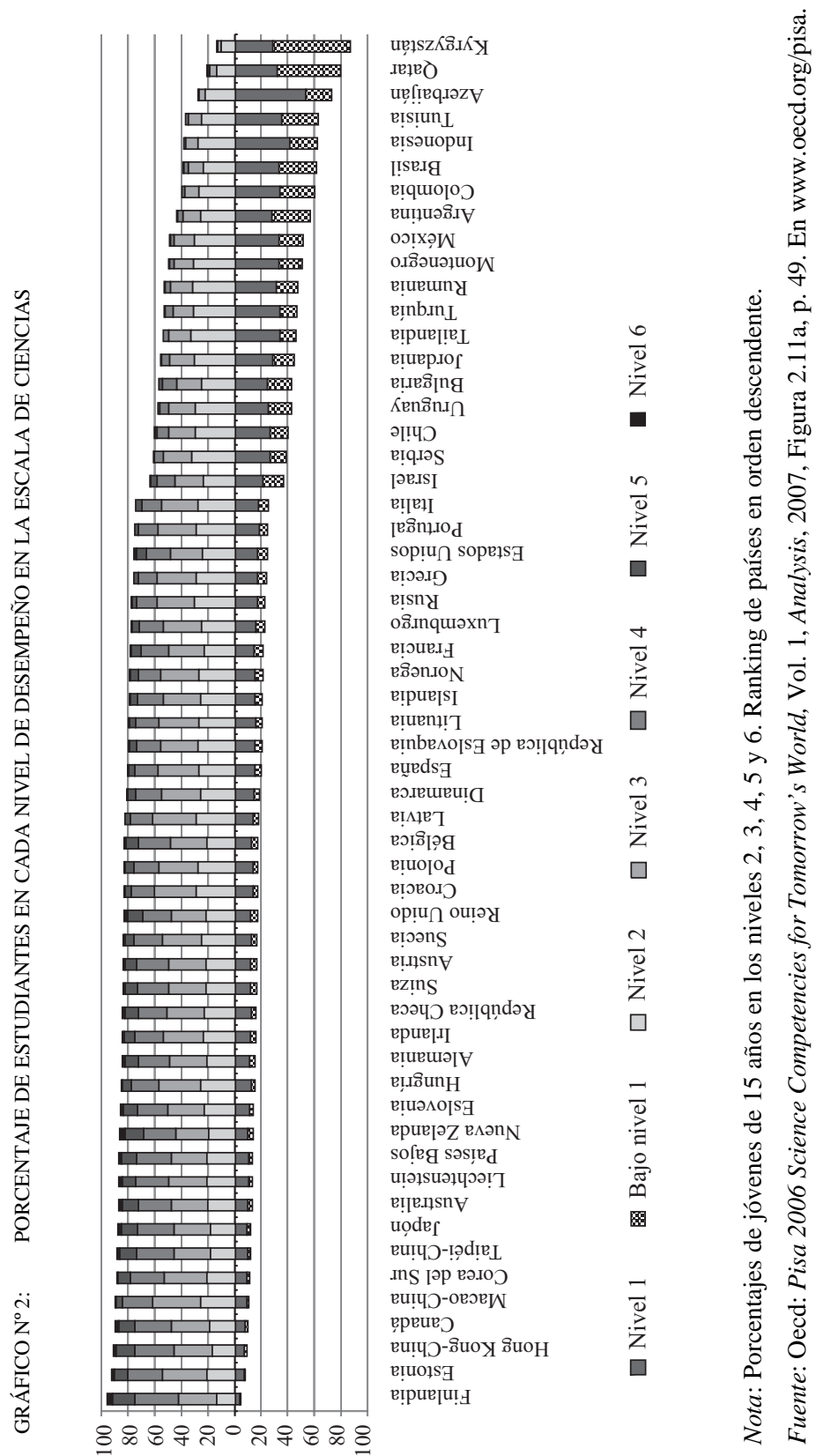
tos científicos básicos a tareas simples y familiares, y/o no pueden presentar explicaciones científicas obvias que se siguen explícitamente de las evidencias entregadas. Por lo que estos jóvenes aparecen con serias desventajas para participar en forma plena en la sociedad y la economía. Y contrasta con los países miembros de la Oecd donde un $5,2 \%$ en promedio se encuentra en esta situación. Finlandia, que obtiene el primer lugar en la escala, no tiene jóvenes con este nivel de desempeño y sólo el 3,6\% de la población alcanza sólo el nivel 1 en contraste con el 26,7\% de la población chilena.

La Oecd (2007) define el nivel 2 como cota base donde los estudiantes comienzan a demostrar las competencias científicas que les permitirán participar activamente en la sociedad y en actividades cotidianas relacionadas con ciencia y tecnología. Si adherimos a este punto de corte podríamos decir que en el nivel 2 los estudiantes se encuentran en el nivel mínimo de manejo del género discursivo que caracteriza la actividad científica. Si se atiende al Gráfico $\mathrm{N}^{\circ} 2$, se aprecia que casi el 60\% de los jóvenes chilenos demuestra ese nivel, y el 29,9\% sólo alcanza esta cota base (nivel de logro 2). Es decir, casi dos tercios de los jóvenes presentan una competencia científica al menos mínima, menos de un tercio presenta sólo mínima y casi un $40 \%$ no alcanza las competencias científicas mínimas, es decir, es prácticamente analfabeto funcional (ubicándose en el nivel 1 o bajo el nivel 1).

Por otro lado, poco menos de un tercio de los jóvenes alcanza el nivel de logro 3, y el 20\% sólo alcanza este nivel de logro, el 8,4\% sólo alcanza el nivel 4, el 1,8\% el nivel 5 y sólo el $0,1 \%$ el nivel superior. Menos de un tercio de los jóvenes chilenos se encuentra entre los niveles 3 y 6 , y menos de un $2 \%$ entre el 5 y 6 (los niveles de mayor desempeño). Esto contrasta con aproximadamente el 56,7\% de los jóvenes de países de la Oecd que alcanza al menos el nivel 3 de desempeño, y el 9\% que alcanza los niveles más altos de desempeño.

Esto es tremendamente preocupante toda vez que según Oecd (2007) los individuos con altos niveles de logro generan relativamente muchas externalidades en creación y utilización de conocimientos en diferentes áreas. Incluso plantea que las investigaciones han mostrado que el efecto en el crecimiento económico del nivel de habilidad en la medición internacional Ials (en la que Chile participó) de una desviación estándar sobre el promedio es alrededor de seis veces mayor que el efecto del nivel de habilidad de una desviación estándar por debajo del 
promedio (Hanushek \& Woessmann, 2007, en Oecd, 2007). Más allá de que estemos mejor que el resto de los países de la región, si nos tomamos en serio las indicaciones de la Oecd (2007) acerca de los estudiantes de mayor desempeño y el impacto en el desarrollo económico y social, tenemos un desafío enorme. La gran mayoría de los estudiantes que tienen más que meras competencias científicas basales identifica sólo los problemas científicos que están claramente descritos; selecciona hechos y conocimientos para explicar fenómenos y aplicar modelos y estrategias de indagación simple; puede desarrollar sólo enunciados breves usando hechos para tomar decisiones en base a conocimientos científicos.

Por último, si miramos el Gráfico $\mathrm{N}^{\circ} 2$ apreciamos que los estudiantes que mejor se desempeñan en Chile no alcanzan los 600 puntos, lo que está muy por debajo de la mayoría de los países Oecd. Más importante aún es el hecho de que las competencias científicas no se distribuyen homogéneamente en la población. En términos generales, en los países de la Oecd tampoco la distribución es homogénea y el nivel socioeconómico y cultural aparece como uno de los factores que más influyen en el desempeño en la escala de ciencias, explicando en promedio un $14,4 \%$ de la varianza del desempeño de los jóvenes en esta escala (Oecd, 2007). Esto indicaría que un factor relevante para manejar el género discursivo propio de la ciencia son las condiciones familiares. Más aún, que este género discursivo se asocia con condiciones familiares favorables en términos socioculturales. Es decir, se podría pensar que esta forma de pensar y hablar atraviesa con mucha más fuerza las familias que tienen índices socioculturales más elevados: mayor escolaridad, menor desempleo, mayor número de bienes familiares, entre otros. Esto es coherente con lo que sugieren los escritos de Vygotski (1934/2001), donde el desarrollo de pensamiento depende estrechamente de qué tanto se ha estado expuesto y se ha sido convocado a hablar y pensar de esa manera por otro. De la misma forma, el fomento de la competencia científica (como de muchas otras competencias) está sujeto a la interacción con un 'otro' competente: si este otro es parte de una minoría de la población, entonces el desarrollo de esta competencia en esa población está en problemas. De hecho, en general el desempeño de los estudiantes de los distintos países es mayor cuando tienen un padre ligado o dedicado a las ciencias. 
Esto también puede ser pensado a nivel país. Los resultados en la escala de ciencias en la prueba Pisa 2006 están fuertemente asociados (no necesariamente de manera lineal) al ingreso per cápita de los países participantes. Los países más ricos obtienen en general mejores rendimientos. Esto pone a Chile en desventaja inmediata con respecto a los países de la Oecd, igual como pone en desventaja a los estudiantes provenientes de contextos socioculturales desaventajados dentro de cada país tendiendo a la inevitable reproducción: mientras menos personas manejen de manera eficiente y sofisticada ese género discursivo, menor es la capacidad de reproducir y desarrollar esa forma de pensar.

Dicho lo anterior, es preciso señalar que en Chile el porcentaje de varianza del desempeño de las estudiantes explicada por factores socioculturales es más alto que en el promedio de países miembros de la Oecd: sobre el 22\% de la varianza del desempeño de ciencias de las jóvenes en esta prueba es explicado por el impacto del índice de nivel socioeconómico y cultural construido por Pisa. En el Gráfico $\mathrm{N}^{0} 3$ se aprecia cómo Chile, junto con Bulgaria y Luxemburgo, es uno de los países en los que el rendimiento en la escala de ciencias depende con más fuerza de las condiciones socioculturales de los estudiantes. Además, Chile y Bulgaria presentan un desempeño promedio bajo el promedio de países Oecd. Interesante es apreciar que Chile se escapa de los países de la región en esta materia. El desempeño de Uruguay, en particular, país que sigue de cerca a Chile en todas las subescalas de la prueba de ciencias, aparece con una asociación menos importante con el nivel sociocultural de los estudiantes (aunque igualmente superior al porcentaje promedio de varianza explicada de los países miembros de la Oecd). Esto también es preocupante, pues mientras en general los jóvenes no cuentan con competencias científicas elevadas, el nivel de desempeño se ve fuertemente asociado al nivel sociocultural de las familias, teniendo el sistema educativo menos impacto y/o capacidad de revertir esta situación.

No obstante lo anterior, es necesario advertir que el desempeño de Chile sólo se explica en parte por las condiciones socioculturales de los estudiantes. Casi el $80 \%$ de la varianza del desempeño de los jóvenes no está explicada por estos factores. De hecho, aun haciendo equivalente el índice económico social y cultural que elabora Pisa, Chile aumentaría sólo en 27 puntos promedio su desempeño en la escala general de ciencias. Es decir, de 438 puntos pasaría a tener 465, lo que 


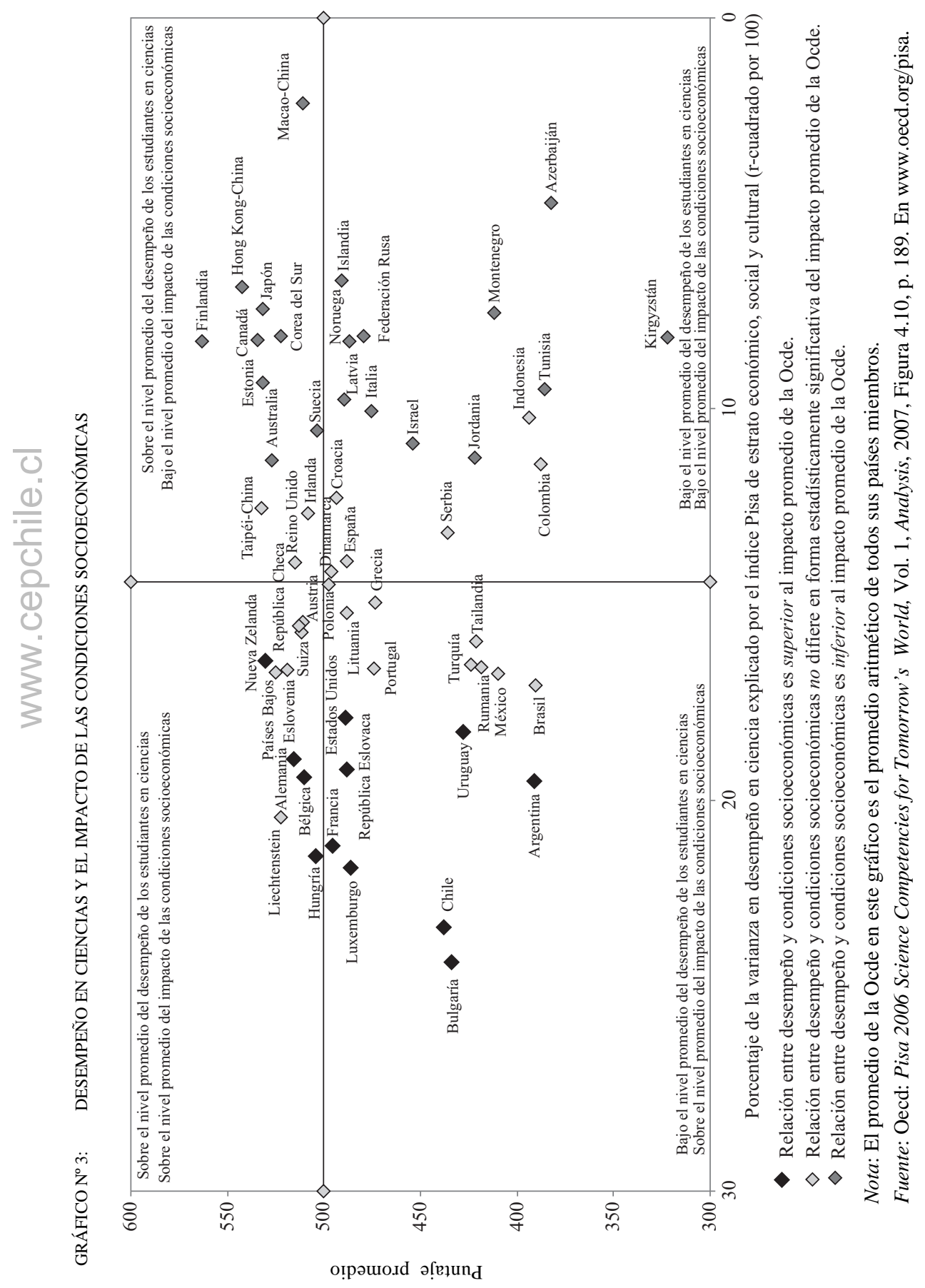


igualmente es un desempeño que deja una brecha importante aún por mejorar (Oecd, 2007).

Otro aspecto muy importante y que se ilustra en el Gráfico $\mathrm{N}^{\circ} 4$ es que aunque Chile no presenta altos niveles de desempeño en alfabetización científica, los jóvenes chilenos sí demuestran mucho interés relativo en estudiar una carrera ligada a las ciencias. Esto hace aún más dramática la inequidad y apunta a un desperdicio enorme de la formación de estos estudiantes y, con ello, de la formación de capital humano en el país. En general, además, los jóvenes chilenos valoran altamente las ciencias (aunque algo menos que el lenguaje y las matemáticas), en comparación con muchos otros países que participaron en la prueba Pisa 2006. Esto quiere decir que aun valorando la ciencia y habiendo muchos jóvenes interesados en seguir carreras científicas, estos jóvenes cuentan con un nivel de alfabetización relativa en ciencias que pone en riesgo sus sueños y proyectos.

En conclusión, en conjunto estos resultados nos indican la debilidad relativa que tiene la población de 15 años en términos de alfabetismo científico con respecto a países de mayor desarrollo económico, siendo la distribución de competencia científica de la población una de las claves de la generación de valor hoy en día. Si, como se discutió, mejorar el alfabetismo en ciencias no es meramente promover el manejo de más contenidos de ciencia y acerca de la ciencia (que por lo demás según la prueba también es una tarea pendiente), sino el manejo de un conjunto de sistemas semióticos y tipos discursivos organizados funcionalmente en el contexto de una actividad social particular (ciencia), el desafío pendiente señala la necesidad de fortalecer todos los caminos a través de los cuales los estudiantes puedan hacerse hablantes de ese género. Por otro lado, nuestro sistema educativo tiene el deber ético de trabajar en este fortalecimiento de manera de promover la equidad del desarrollo social y económico. De lo contrario, estamos desperdiciando recursos muy relevantes en todos aquellos jóvenes que, aun queriendo y pudiendo, no alcanzan niveles de desempeño alto por el poco manejo del género discursivo propio de la ciencia en sus hogares y actividades extraescolares.

Particularmente, el desarrollo de la argumentación en la escuela parece ser uno de estos caminos. Por un lado, es un tipo discursivo propio del pensamiento científico y que moviliza los conocimientos en ciencia en muchas de sus aplicaciones. De hecho, una de las claves de 


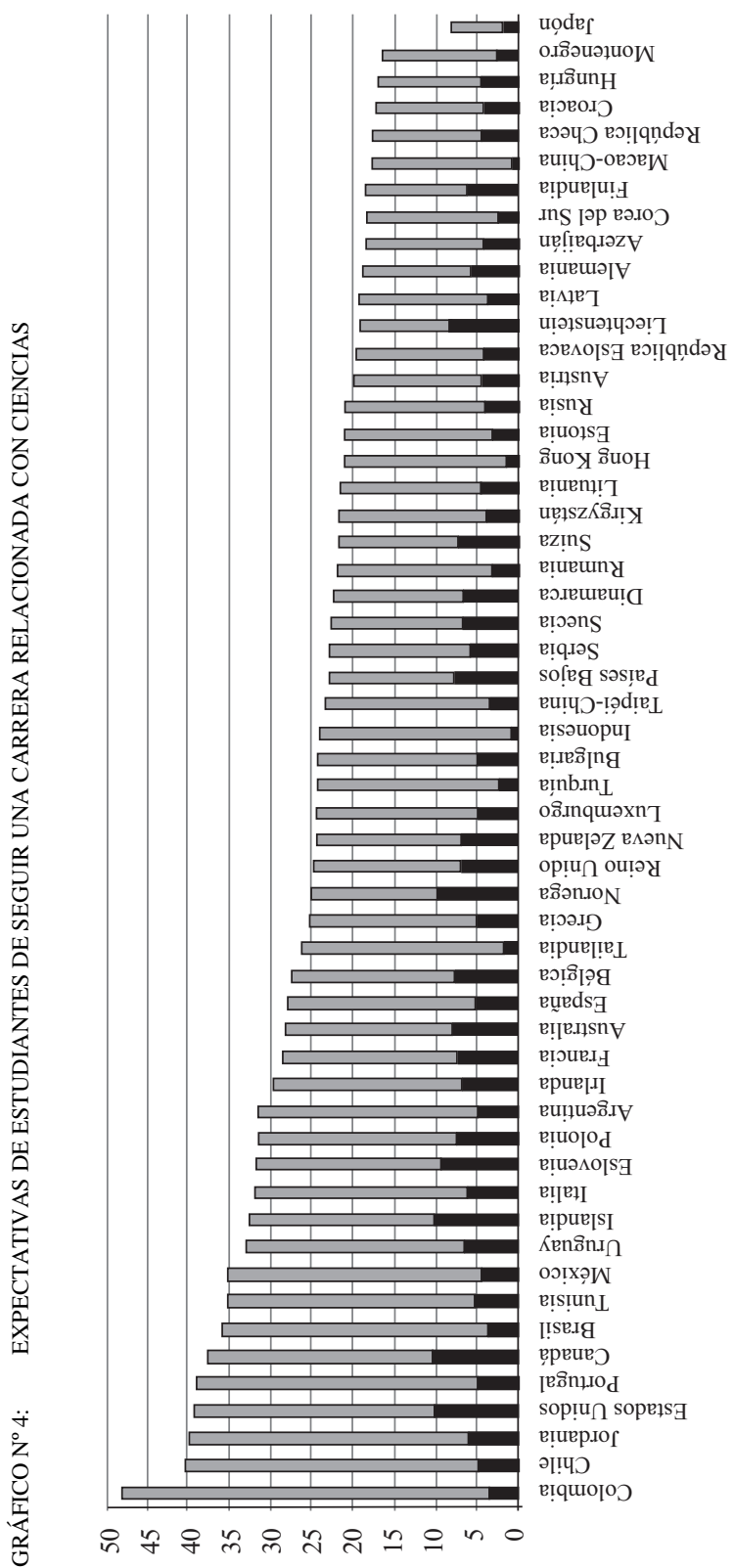


la ciencia es crear condiciones en las cuales los hallazgos puedan ser comunicados como tales y aceptados por la comunidad científica. Por otro lado, la argumentación es un tipo discursivo que los jóvenes utilizan diariamente en su vida cotidiana, por tanto puede ser una alternativa interesante para acercar el discurso 'científico' a los jóvenes independientemente de su proveniencia y nivel socioeconómico.

\section{Argumentación}

La argumentación es un componente esencial de la comunicación humana. La capacidad de apoyar y contrarrestar ideas es fundamental para la vida social. Las habilidades de elaborar puntos de vista con respecto a los objetos del mundo y defender esos puntos de vista ante posibles visiones alternativas (o ideas que contradicen e incluso critican posiciones que están siendo defendidas), son centrales para la comprensión del mundo natural y social. La argumentación, entonces, no pertenece sólo al ámbito de la lógica y tampoco puede ser entendida sólo como un dispositivo discursivo que aparece en actividades sociales específicas como los debates (Toulmin, 1958; Van Eemeren, Grootendorst y Snoeck, 1996; Perelman \& Olbrecht-Tyteca, 1969).

De hecho, es de creciente consenso la importancia que tiene el desarrollo de habilidades argumentativas en la promoción del pensamiento y la construcción de conocimiento (Leitão, 2000; Kuhn, 1992; Billig, 1987; Pontecorvo, 1988; Pontecorvo \& Pirchio, 2000; Forman, 2000; Rojas-Drummond \& Peon Zapata, 2004; Yackel, 2001). Diversos autores enfatizan que la argumentación favorece el cambio de perspectivas (Leitão, 2000: Orsolini \& Pontecorvo, 1992; Pontecorvo, 1988, Forman, Larreamendy-Joerns, Stein \& Brown, 1998). Este aspecto es importante, pues sugiere que para la construcción de conocimiento es necesario un otro, un punto de vista alternativo. De esta manera se configura como un proceso de pensamiento conjunto en el que la perspectiva del otro tiene un efecto inmediato sobre la propia, ya sea para hacer evidente sus aspectos débiles o para abrir paso a otra alternativa en que se puede pensar el mismo asunto. El espacio de negociación que abre la argumentación favorece una mirada creativa, divergente, y el desarrollo de procesos de pensamiento complejos a través de los cuales se realiza una construcción de conocimiento con- 
junta (reconstrucción progresiva del conocimiento de cada individuo). En otras palabras, argumentar promueve el desarrollo cognitivo (Leitão, 2000 y 2003; Kuhn, 1992, 1999; Rogoff, 1993).

Ahora, tanto Leitão (2003; 2002), Kuhn (1992; 1999) como Mason \& Santi (1994) enfatizan un segundo aspecto epistémico propio de la argumentación: su potencial meta-cognitivo. En la medida en que argumentar favorece en los individuos la reconstrucción de perspectivas sobre los fenómenos del mundo, favorece la reflexión sobre los fundamentos del conocimiento que ellos producen. Leitão (2002) plantea que la posibilidad de meta-cognición está dada por los movimientos propios de la argumentación y sus respectivos mecanismos semióticos. Es decir, cada movimiento (argumento, contra-argumento y respuesta) promueve un proceso epistémico específico. De esta manera, si bien la elaboración de un argumento implica un pensamiento meta-cognitivo incipiente, es el contra-argumento el movimiento que gatilla el paso del pensamiento a un funcionamiento meta-cognitivo como tal, pues fuerza al individuo a mirar su punto de vista desde otro alternativo que es articulado explícitamente, con el fin de defenderlo o llegar a un consenso. En consecuencia, la meta-cognición puede alcanzar distintos niveles de elaboración según la estructura argumentativa que se realice (Mason \& Santi, 1994; Larraín, 2007). Esto es relevante, pues no da igual el tipo y calidad de argumentación en términos de desarrollo cognitivo: si bien involucrarse en argumentación siempre traerá de la mano construcción de conocimiento, este proceso puede ser de distintos grados de elaboración y complejidad según como se argumente.

La investigación en la relación entre argumentación y metacognición sugiere que la primera promueve un tipo de pensamiento reflexivo, crítico y creativo, que es fundamental para la apropiación de contenidos curriculares. La construcción y reconstrucción de perspectivas que acompañan la argumentación promueven un tipo de aprendizaje de los contenidos curriculares socio-constructivo, es decir, donde más que memorizar y repetir, lo que el niño, niña o joven hace es procesar los contenidos de manera de comprenderlos o de incorporarlos a su sistema conceptual. Esto trae como consecuencia, más que un almacenamiento de contenidos, un desarrollo cognitivo y conceptual que no se olvida, que permite continuar construyendo conocimiento sobre esta nueva base. Aprender a argumentar no sólo facilita el aprendizaje de los contenidos actuales sino que es aprender una habilidad 
para la vida: la enseñanza de habilidades argumentativas es a la vez promoción de habilidades de pensamiento 'superior'.

En este contexto, la argumentación se sitúa dentro de aquellas habilidades que son sindicadas como fundamentales en una sociedad como la actual donde el capital humano es central, el conocimiento es el bien más preciado y el manejo flexible de la información oral y escrita (alfabetización) una competencia básica. De hecho, no es coincidencia que la prueba internacional Pisa 2000, 2003 y 2006, que busca medir el grado de alfabetización funcional, es decir, las destrezas en el manejo de la información que tienen jóvenes de 15 años en lenguaje, matemáticas y ciencias, defina para cada uno de estos ámbitos disciplinarios las categorías de evaluación más elevadas incorporando la capacidad de argumentar (Oecd, 2004; Mineduc, 2004a). En el caso de ciencias, se enfatiza la habilidad de elaborar cadenas de razonamiento, evaluar puntos de vista alternativos o diferentes perspectivas, y comunicar argumentos científicos con detalle y/o precisión; en el caso de matemáticas se requiere la habilidad de encadenar razonamientos y la habilidad para argumentar, justificar y comunicar los resultados; en el caso de lectura se establece la habilidad de evaluar críticamente y de manejar ideas y opiniones distintas de las sugeridas por la lectura.

En síntesis, si las competencias fundamentales que la escolaridad debe desarrollar se relacionan con habilidades que reflejen desarrollo cognitivo avanzado y meta-cognición (pensar, elaborar, criticar, discriminar, crear y producir nueva información) que asegure que el individuo podrá, fuera de la escuela, cumplir con las demandas mínimas de la sociedad, la argumentación aparece como una habilidad fundamental a desarrollar en la etapa de escolarización (Oecd, 2001).

\subsection{Argumentación y ciencias}

Ahora, si bien por lo que hasta aquí se ha discutido las habilidades argumentativas parecieran ser importantes para el aprendizaje de contenidos curriculares de todas las disciplinas, éstas son especialmente importantes en el caso de ciencias (Erduran, Ardac \& YakmaciGuzel, 2006; Millwood, 2006). En el género discursivo propio de la ciencia predomina el tipo discursivo argumentativo, toda vez que los hallazgos no son evidentes en sí mismos sino que dependen de una 
estructura argumental para ser reconocidos como tales. Esta estructura argumental incluye reconocer el problema científico involucrado, hipotetizar, diseñar experimentos que puedan responder las distintas preguntas, discutir los resultados contrastando evidencia, reconocerlos como hallazgos ligándolos a las evidencias, tomar posición con respecto a éstos, divulgarlos y convencer al resto de la comunidad (Hammer, Russ, Mikeska \& Scherr, in press; Duschl \& Ellenbogen, 1999). Incluso la necesidad del manejo de la competencia científica para los ciudadanos que no se dedicarán a la ciencia implica como mínimo poder seguir un argumento y reconocer las potenciales debilidades de éstos. Esto es central, por ejemplo, para seguir las indicaciones médicas, tomar decisiones económicas, seguir distintos estilos de alimentación, de tratamiento psicológico, entre muchos otros.

Por otro lado, considerando que la argumentación es transversal al tipo de habla cotidiano de una población (al menos en ciertos niveles mínimos), puede facilitar la cercanía de los estudiantes con el pensamiento científico, que en sí mismo muchas veces les es extremadamente ajeno. Invitar a la ciencia por la vía de estimular el debate y la defensa de una postura puede ser una buena estrategia: por un lado se potencian habilidades argumentativas y con ellas habilidades de pensamiento profundo (que les servirán más allá de las ciencias) y, por otro, se promueve el desarrollo del manejo de la estructura funcional propia del género de habla científico, en la cual la argumentación si bien es central se conjuga con otros recursos como conocimiento de las ciencias, valoración de la ciencia, entre muchos otros.

De hecho, sumado a lo anterior está el hecho de que la familiaridad con el discurso científico no está equitativamente distribuida en la población, pues distancia más a los niños y jóvenes de escasos recursos que provienen de hogares y comunidades donde incluso el lenguaje cotidiano es precario (en comparación con familias más escolarizadas). Esto fuerza a considerar la entrada al tipo de habla propio de la ciencia a través de tipos de discurso que sean más familiares. Ballenger (1997), a través de un estudio con niños haitianos, plantea que una de las causas del fracaso escolar en estas materias es la incoherencia entre el lenguaje de la familia y el lenguaje en el aula de ciencias. Si bien esto había sido planteado ya por Heath (1982), en un estudio que muestra cómo la coherencia del tipo de habla familia-escuela de los estratos socio-económicos más favorecidos determina el éxito en la 
alfabetización funcional de los niños, en ciencias no había sido mencionado. Ballenger (1997) muestra cómo el argumentar en las aulas de ciencia ayuda a los niños más desaventajados a involucrarse en su aprendizaje.

\subsection{Habilidades argumentativas y enseñanza de la argumentación en Chile}

Hoy en Chile no existen estudios que den cuenta de las habilidades argumentativas de los estudiantes y de la forma como la escuela promueve el desarrollo de esta habilidad. Sin embargo, Chile participó en los estudios internacionales Pisa 2000 y 2006, los que, como se comentó, indirectamente miden habilidades argumentativas en la medida en que éstas son necesarias para alcanzar los niveles mayores en las tres áreas medidas: matemáticas, lectura y ciencias. Como ya se comentó, la información que provee esta medición no es alentadora. En promedio los estudiantes chilenos muestran un nivel de alfabetización marcadamente menor que el promedio de países Oecd. Más aún, sólo un $0,5 \%$ de estudiantes chilenos alcanza el nivel más alto de alfabetización en lectura, en ciencias sólo un 5\% de la población supera, en promedio, los 574 puntos, y en matemáticas sólo un 5\% supera los 532 puntos. Esto quiere decir que los estudiantes chilenos muestran tener pocas habilidades de pensamiento en general y, en particular, escasas habilidades argumentativas para el manejo de los respectivos lenguajes disciplinarios, en particular.

Esto es concordante con los resultados de la prueba que mide calidad de argumentación desde el 2003 en los estudiantes que ingresan a la Pontificia Universidad Católica de Chile. Los resultados del test tomado en 2004 y 2005 muestran que la calidad argumentativa del texto en promedio se encuentra entre un desempeño con algunas limitaciones y un desempeño aceptable, y la habilidad central en el desarrollo de meta-cognición, la de elaborar puntos de vista alternativos, se encuentra subdesarrollada: en promedio los estudiantes de todas las carreras tuvieron entre un desempeño deficiente y un desempeño con algunas limitaciones (Manzi \& Flotts, 2008). Aunque en 2006 tanto la calidad de argumentación como la elaboración de puntos de vista subieron y se situaron entre desempeño aceptable y buen desempeño, esta última habilidad aún aparece subdesarrollada en comparación con los aspectos de contenido (calidad de la argumentación, tesis y evaluación 
global de la calidad del texto). Esto cambió en 2007 y 2008, cuando la contra-argumentación fue levemente más alta que la evaluación global de la calidad de la argumentación. Esto muestra que el nivel argumentativo de estudiantes que egresan de enseñanza media y son aceptados en esta universidad (que recibe a estudiantes de alto desempeño) es relativamente bajo, siendo medianamente capaces de involucrarse en actividades meta-cognitivas.

Más aún, los resultados de este test muestran que existe una alta correlación con las notas obtenidas en el primer semestre de desempeño universitario, lo que sustenta la tesis de la relación entre argumentación y calidad de aprendizaje y rendimiento académico. Lo más sorprendente es que el desempeño de los estudiantes de carreras de las áreas de ciencias exactas (matemáticas, construcción civil, licenciatura en física, licenciatura en química) es sistemáticamente menor que el desempeño de los jóvenes en carreras ligadas a áreas humanistas y medicina. Digo sorprendente, pues, aunque es esperable que los estudiantes que entran a carreras humanistas tengan mayores aptitudes y motivación para la escritura en general, y en particular para habilidades de argumentación, esto no es funcional a la alfabetización científica. Aun considerando que la argumentación en ciencias exactas incluye otros sistemas semióticos distintos del lenguaje verbal que no son medidos por la prueba, las habilidades argumentativas verbales debieran ser promovidas por la escolaridad que está estrechamente ligada al quehacer científico.

Esto sindica la debilidad del sistema escolar en promover el desarrollo de pensamiento avanzado y flexible en general, y lo caracteriza como un sistema que prepara principalmente para la adquisición de conocimientos de manera rígida y apegada a los contextos en los que éstos fueron aprendidos. A la vez, estos resultados deben hacernos reflexionar sobre la necesidad de promover tempranamente habilidades de pensamiento acerca de lo científico que permitan el avance en alfabetización científica. La argumentación, a mi parecer y según lo expuesto hasta aquí, no sólo es una habilidad más entre otras de pensamiento que componen la alfabetización científica, sino probablemente es una habilidad discursiva estratégica para el desarrollo de pensamiento científico, toda vez que pensar científicamente involucra argumentar en algún nivel, y que la argumentación, además de gatillar procesos de pensamiento superior (meta-cognitivos), favorece el pro- 
ceso mismo de construcción de conocimientos y, con eso, el grado en que éstos serán apropiados por parte de los estudiantes.

Por su parte, aunque no existen estudios en Chile que den cuenta directamente de la forma como se enseña a argumentar en las aulas, datos de otros estudios acerca del discurso docente pueden orientar algunas reflexiones al respecto. Preiss (2007) muestra que el discurso docente es similar al de las aulas de Estados Unidos, donde la mayor parte del tiempo habla el profesor, donde la mayor parte del habla del profesor está destinada a cuestiones disciplinarias y de organización, y el habla dedicada al contenido está básicamente destinada a entregar información - a diferencia de Japón, donde el habla del docente es mayoritariamente de entrega de contenidos y donde los docentes promueven la construcción de argumentos colectivos y el involucramiento en reflexión y evaluación (Inagaki, Morita, Hatano, 1999)—. Preiss (2007) estudió el tipo de preguntas y seguimiento que hacen profesores en los primeros años de enseñanza básica en clases de lenguaje. Sus resultados muestran que las preguntas que incitan a la elaboración por parte de los estudiantes son mínimas y los seguimientos que hacen a las respuestas de los estudiantes prácticamente nunca reformulan. Es decir, los docentes en la educación básica sostienen el tipo de secuencia discursiva típica según Wertsch (1998): Iniciación-respuesta-evaluación (I-R-E). Este tipo de discurso es claramente poco argumentativo.

En síntesis, las pocas mediciones que dan cuenta de la habilidad argumentativa de los estudiantes chilenos muestran datos inquietantes, sobre todo si se piensa en la relación entre argumentación y calidad de aprendizajes curriculares y desarrollo de habilidades de pensamiento superior. Especialmente los bajos niveles de esta habilidad y el bajo desempeño que muestran los estudiantes chilenos en contenidos curriculares de ciencia en educación básica y alfabetización en el área, fuerzan a poner las esperanzas en que un aumento en habilidades argumentativas pueda promover mejorías en estos aspectos, y a través de ellos, en el desarrollo de competencias intelectuales fundamentales para desenvolverse en la sociedad actual.

\section{Palabras finales}

La argumentación no se desarrolla espontáneamente. Aunque la argumentación empieza a desarrollarse cuando el niño comienza a hablar, el grado en que integre este tipo discursivo dependerá del tipo y 
cantidad de interacción conversacional argumentativa que sostenga con otros. Aquí la educación formal ocupa un lugar privilegiado para el aprendizaje de la argumentación por la sistematicidad de las interacciones discursivas que el niño, niña o joven tiene con sus pares y profesores (Vygotski, 1934/2001).

De hecho, diversos estudios muestran cómo la educación formal en el segundo ciclo básico (niños entre diez y catorce años) puede hacer una diferencia en el desarrollo de habilidades argumentativas cuando los niños participan en grupo en discusiones curriculares (Kuhn \& Udell, 2003), en particular cuando las discusiones en grupos pequeños son mediadas por el profesor (De Chiaro \& Leitão, 2005), cuando se enseña explícitamente a argumentar a los estudiantes (Rojas-Dummond \& Peon, 2004), o cuando el profesor media los aportes individuales de niños en discusiones de la clase completa, por ejemplo, haciéndoles preguntas que requieran elaboración (Hammer et al., en prensa). Es preciso señalar que todos los niños adquieren algunas habilidades argumentativas, pero si la escuela no incluye sistemáticamente actividades para su desarrollo, estas habilidades van a depender únicamente de factores individuales y familiares, por lo que habrá grandes diferencias entre los jóvenes, y es probable que el nivel de habilidades argumentativas en términos generales sea muy insuficiente (sobre todo si esto se cruza con factores socioeconómicos). De hecho un estudio de Scholtz, Braund, Hodges, Koopman, \& Lubben (2008), donde se midió la habilidad argumentativa espontánea de 266 estudiantes de educación media de colegios sudafricanos, encontró que el $85 \%$ de los estudiantes presentaba niveles básicos de argumentación (de cinco niveles donde el 1 era el más precario y el 5 el más avanzado, estos estudiantes se ubicaron entre los niveles 1 y 2).

Dicho lo anterior, si es importante fortalecer la enseñanza y el aprendizaje de ciencias, ya sea porque hoy este aprendizaje en Chile no alcanza logros esperados o porque el desarrollo de pensamiento científico es relevante para el desarrollo del capital humano del país, entonces enseñar a argumentar es una alternativa que hay que atender (Osborne, Erduran, Simon, \& Monk, 2001). Por lo demás, más que enseñar a argumentar en general, es necesario enseñar a argumentar en ciencias. 


\section{REFERENCIAS BIBLIOGRÁFICAS}

Bajtín, Mikhail: “El Problema de los Géneros Discursivos”. En Estética de la Creación Verbal. Buenos Aires: Siglo XXI Editores, 2002.

Ballenger, Cynthia: "Social Identities, Moral Narratives, Scientific Argumentation: Science Talk in a Bilingual Classroom”. En Language and Education 11, 1997.

Billig, Michael: Arguing and Thinking: A Rhetorical Approach to Social Psychology. Cambridge: Cambridge University Press, 1987.

De Chiaro, Sylvia y Selma Leitão: "O Papel do Profesor na Construcão Discursiva da Argumentacão em Sala de Aula”. En Psicologia: Reflexão e Crítica 18 (3): 350-357, 2005.

Duschl, Richard A. y Kirsten Ellenbogen: "Middle School Science Students' Dialogic Argumentation”. En Michael Komorek et al. (eds.), Proceedings: Second International Conference of ESERA: Research in Science Education. Past, Present and Future 2: 420-423, Kiel, IPN, 1999.

Erduran, Sibel, Dilek Ardac y Buket Yakmaci-Guzel: "Learning to Teach Argumentation: Case Studies of Pre-service Secondary Science Teachers”. En Eurasia of Mathematics, Science and Technology Education 2, 2006.

Eyzaguirre, Bárbara y Carmen le Foulon: "La Calidad de la Educación Chilena en Cifras”. En Estudios Públicos 84 (primavera, 2001).

Forman, Ellice A.: "Knowledge Building in Discourse Communities". En Human Development 43: 364-368, 2000.

Forman, Ellice A., Jorge F. Larreamendy-Joerns, J. Mary Kay Stein y Catherine Brown: “'You're Going to Want to Find out Which and Prove It': Collective Argumentation in Mathematics Classroom”. En Learning and Instruction 6: 527-548, 1998.

Hammer, David, Rosemary Russ, Jamie Mikeska et al.: "Identifying Inquiry and Conceptualizing Students’ Abilities”. En Richard Duschl \& Richard Grandy (eds.), Establishing a Consensus Agenda for K-12 Science Inquiry. Rotterdam, NL: Sense Publishers, 2008.

Haye, Andrés: "Living Being and Speaking Being: Toward a Dialogical Approach to Intentionality”. En Integrative Psychological and Behavioral Science 42: 157-163, 2007.

Heath, Shirley Brice: "What no Bedtime Stories Jeans. Narrative Skills at Home and School”. En Language in Society 11: 49-76, 1982.

Inagaki, Kayakoyo, Eiji Morita y Giyoo Hatano: “Teaching and Learning of Evaluative Criteria for Mathematicals Arguments Through Classrooms Discourse: A Cross National Study”. En Mathematical Thinking and Learning 1: 93-111, 1999.

Kuhn, Deanna: “Thinking as Argument”. En Harvard Educational Review 62: 155178, 1992.

"Metacognitive Development”. En Lawrence Balter y Catherine Tamis-Le Monda (eds.), Child Psychology. A Handbook of Contemporary Issues. New York: Psychology Press, 1999.

“Metacognitive Development”. En Current Directions in Psychological Science 9: 177-181, 2000. 
Larrain, Antonia: “Alfabetismo Funcional: Un Desafío de Todos”. En Psykhé 11 (1): 43-56, 2002.

"Condiciones Retóricas y Semióticas de la Auto-Argumentación Reflexiva". Tesis presentada a la Pontificia Universidad Católica de Chile como parte de los requisitos para optar al grado de Doctor en Psicología, 23 de abril, 2007.

Laugksch, Rüdiger C.: "Scientific Literacy: A Conceptual Overview”. En Science Education 84: 71-94, 2000.

Leitão, Selma: "The Potential of Argument of knowledge Building”. En Human Development 43: 332-360, 2000.

"Self-Argumentation: The Rhetorical Dimension of Meta-Cognition". Trabajo presentado en Kitchen Seminar de Francis Hiatt School of Psychology, Clark University, MA. Noviembre, 2002.

"Evaluating and Selecting Counterarguments. Studies of Children's Rhetorical Awarness”. En Written Communication 20: 269-303, 2003.

Manzi, Jorge y Paulina Flotts: "Medición de Habilidades de Comunicación Escrita: Hallazgos y Proyecciones”. Trabajo presentado en coloquio MIDE UC, 27 de octubre 2008. Extraído de www.mideuc.cl el 12 de diciembre de 2008.

Mason, Lucia y Marina Santi: “Argumentation Structure and Metacognition in Constructing Shared Knowledge at School”. Trabajo presentado en la Reunión Anual de la American Educational Research Association, New Orleans, April 1994. Extraído de http://eric.ed.gov/ERICDocs/data/ericdocs2/content_ storage_01/0000000b/80/26/c1/d0.pdf el 27-01-2007.

Millwood, Kelli A: “A Comparison of Students' Conceptions about Nature of Argumentation in School and Professional Science”. International Conference on Learning Sciences Proceedings of the 7th International Conference on Learning Sciences. Bloomington, Indiana, 962-963, 2006.

Mineduc: La Educación Chilena en el Cambio de Siglo: Políticas, Resultados y Desafíos. Informe nacional de Chile, Oficina Internacional de Educación Unesco, 2004a.

- Chile y el Aprendizaje de Matemáticas y Ciencias según Timss. Resultados de los Estudiantes Chilenos de $8^{\circ}$ Básico en el Estudio Internacional de Tendencias en Matemáticas y Ciencias 2003. Unidad de currículo y evaluación, 2004b. Extraído de www.simce.cl el 10 de noviembre de 2008.

- Rendimiento de Estudiantes en Ciencia, Lectura y Matemáticas. Informe nacional de Chile, 2008.

- Competencias para la Vida. Resultado de los Estudiantes Chilenos en el Estudio Pisa 2000. Ministerio de Educación, Unidad de Currículum y Evaluación, 2001.

Oecd: Literacy in the Information Age: Final Report of the International Adult Literacy Survey. París y Ottawa: Statistics Canada, 2000.

- Literacy Skills for the World of Tomorrow. Further Results from PISA 2000. Institute for Statistics Unesco, 2001.

- Marcos Teóricos de PISA 2003: La Medida de los Conocimientos y Destrezas en Matemáticas, Lectura, Ciencias y Resolución de Problemas. Madrid: Ministerio de Educación y Ciencia, Instituto Nacional de Evaluación del Sistema Evaluativo (Inecse), 2004. 
PISA 2006. Marco para la Evaluación. Conocimientos y Habilidades en Ciencias, Matemáticas y Lectura. Madrid: INCE, 2006. Extraído de http:// www.ince.mec.es/marcosteoricospisa2006.pdf.

PISA 2006. Science Competencies for Tomorrow Worlds. Vol. 1. Analysis. Oecd 2007. www.Oecd/pisa.org.

Orsolini, Marguerita y Clotilde Pontecorvo: “Children's Talk in Classrooms Discussions”. En Cognition and instruction 9: 113-136, 1992.

Osborne, Jonhatan, Sibel Erduran, Shirley Simon y Martin Monk: "Enhancing the Quality of Argument in School Science”. En School Science Review 82 (301): 63-70, 2001.

Perelman, Chaim y Lucie Olbrechts-Tyteca: Tratado de la Argumentación. La Nueva Retórica. Madrid: Gredos, 1969.

Pontecorvo, Clotilde: "Discussion for Reasoning. The role of Argument in Knowledge Construction”. En Erik de Corte, H. Lodewijks, R. Parmentier \& P. Span (organizadores), Learning and Instruction. A Publication of the European Association for Research of Learning and Instruction. Leuven, Belgium: Leuven University Press, 1998.

Pontecorvo, Clotilde y Sabine Pirchio: “A Developmental View on Children's Arguing: The Need of the Other”. En Human Development 43: 361-363, 2000.

Preiss, David: “The Chilean Instructional Pattern for the Teaching of Language: A Video-Survey Study Based on a Natural Program for the Assessment of Teaching”. En Learning and Individual Differences, 19:1-11, 2009.

Rogoff, Barbara: Aprendices del Pensamiento. Barcelona: Paidós, 1993.

Rojas-Drummond, Sylvia y Margarita Peon Zapata: "Exploratory Talk, Argumentation and Reasoning in Mexican Primary School Children”. En Language and Education 18, 2004.

Scholtz, Zena, Martin Braund, Merle Hodges, Robert Koopman y Fred Lubben: "South African Teachers' Ability to Argue: The Emergence of Inclusive Argumentation”. En International Journal of Educational Development 28 (1): 21-34, 2008

Sjфberg, Svein. "Scientific Literacy and School Science. Arguments and Second Thoughts”. En Svein Sjфberg and Egil Kallerud (eds.), Science, Tachnology and Citizenship. Oslo: NIFU, 1997.

Toulmin, Stephen: The Uses of Argument. Cambridge: Cambridge University Press, 1958.

Van Eeemeren, Franz H., Rob Grootendorst y Francisca Snoeck: Fundamentals of Argumentation Theory: A Handbook of Historical Backgrounds and Contemporary Developments. Mahwah: Lawrence Erlbaum, 1996.

Vygotski, Lev S.: "Pensamiento y Lenguaje”. En Obras Escogidas II. Madrid: Visor, [1934]/2001.

Wertsch, James: La Mente en Acción. Buenos Aires: Aique, 1984.

Yackel, Erna: "Explanation, Justification and Argumentation in Mathematics Classrooms". Proceedings of the $25^{\text {th }}$ Conference of the International Group of Psychology, 2001. 